\title{
Paraspinal muscle hypotrophy and chronic discogenic low back pain
}

\author{
Aleksandra Truszczyńska-Baszak ${ }^{1,2}$, Bruno Krauze $^{1}$, Adam Tarnowski $^{3}$ \\ ${ }^{1}$ Faculty of Rehabilitation, Józef Pilsudski University of Physical Education, Warsaw, Poland; 2 Spine Surgery Department, \\ Prof. A. Gruca Independent Public Clinical Hospital, Otwock; ${ }^{3}$ Faculty of Psychology, Warsaw University, Warsaw, Poland
}

\section{Summary}

Study aim: Low back pain is accompanied by deconditioning of trunk muscles due to pain limiting patients' physical activity, but so far it has not been explained whether the changes in the structure of muscles are the cause of disc disease or its result. The aim of the study was to analyze the prevalence of segmental paraspinal muscle hypotrophy in patients with chronic low back pain and sciatica.

Material and methods: The study involved magnetic resonance imaging (MRI) of 40 patients: 20 women and 20 men aged from 30 to 47, mean $39.51 \pm 3.73$ years, with single level disc extrusion at L4-L5. The entire cross-sectional area of the paraspinal muscles, the adipose tissue area in the paraspinal muscles and the extensor muscle tissue area at the level of L4-L5 were measured and compared with the healthy L3-L4 level. T2-weighted axial slices were used to facilitate distinguishing between the muscle and the fat tissue.

Results: Fat tissue ingrowth and paravertebral muscle tissue hypotrophy at the disc extrusion level were highly statistically significant $(\mathrm{p}<0.001)$ compared to the healthy level.

Conclusions: 1 . Ingrowth of the adipose tissue into the muscle tissue occurs only at the level of disc extrusion. 2. It seems reasonable to introduce strengthening exercises after the resolution of pain in order to rebuild the muscles of the spine.

\section{Key words: MRI - Muscular hypotrophy - Lumbosacral region/physiopathology - Muscular hypotrophy - Diagnosis}

\section{Introduction}

The prevalence of low back pain during the whole life and cost of its treatment are rising with the aging of populations $[15,16,23]$. The causes of pain are related primarily to the impairment of the mutual relation between the intervertebral disc and the vertebral canal $[5,7]$. In recent years, attention has been paid to the problem of the "deconditioning" of the trunk muscles in the course of disc disease. This condition may be a result of pain limiting patients' physical activity, but so far it has not been explained whether changes in the structure of muscles are the cause of disc disease or its result [16]. Some reports have shown a small or non-significant relationship between muscle strength, endurance, MRI changes and low back pain [20], while others have proved that the trunk muscles in patients with back pain were significantly weaker than in healthy subjects [14], and unilateral lumbar multifidus hypotrophy was often associated with back pain [6]. Structural changes within the multifidus and the erector spinae have also been reported in patients undergoing surgery due to sciatica [12]. Hypotrophy of these muscles was characterized in the magnetic resonance imaging (MRI) image by a reduction in the cross-sectional area of the muscles. There were also observed "fat deposits", as well as muscle fiber hypotrophy in the histopathological examination.

A review of the literature indicated that the authors in the analysis of imaging examinations focused first on the measurements of the muscles' cross-sectional area (CSA) as a measure of their strength [19]. Subsequent works have suggested, however, that the CSA changes correlated only to a small extent with the muscle function, because the muscle tissue quality was not taken into account [21]. Therefore, attention was then focused on the muscle quality and observations concerning the adipose tissue ingrowth into the paraspinal muscles [17], as well as the qualitative assessment [13] and quantitative evaluation of the muscle tissue $[6,8]$.

Nevertheless, many of these studies were based on groups composed of a few subjects with a non-specific diagnosis or they were qualitative in nature, and therefore 
the value of these studies in relation to people with back pain and sciatica caused by disc extrusion is limited.

The aim of the study was to analyze the prevalence of muscle hypotrophy, including the reduction in the total cross-sectional paraspinal areas, the cross-sectional muscle area and the cross-sectional adipose tissue area, in patients with chronic discogenic back pain and sciatica. It was assumed that patients with disc disease would show, at the level of the damaged intervertebral disc, clear signs of muscle tissue infiltration by the adipose tissue compared to the adjacent level of the healthy intervertebral disc.

\section{Material and methods}

The study was approved by the responsible institutional ethical review board. The study included 40 patients, out of 1071 hospitalized (due to various spinal problems) patients, who met the inclusion criteria. Details are in presented in a flow chart (Fig. 1). In the study group, there were 20 women and 20 men aged from 30 to 47, mean $39.51 \pm 3.73$ years, with disc disease of the lumbar spine. All analyzed patients suffered from chronic back pain with duration over three months, mean $8.34 \pm 2.16$ months, sciatica and neurological deficits: motor weakness and sensory loss from the L5 nerve root.

The inclusion criteria for the study were as follows: low back pain and sciatica lasting over three months due to disc extrusion confirmed by MRI, changes at a single level (only at L4-L5), absence of other diseases affecting the locomotor system, low to moderate physical activity level.

The exclusion criteria were as follows: changes in segments other than L4-L5, a history of previous spinal surgery, scoliosis, spinal canal stenosis or spondylolisthesis, degenerative changes in facet joints, serious spinal pathology (tuberculosis, inflammation, metastasis, fracture), professional sport training.

The tests consisted in measuring the fat area in the paravertebral muscles at the level of L4-L5 and comparing it to the fat tissue area at a healthy level of L3-L4. MR images that were available in the system Carestream Health Solution version 11.0-pacs01 were used for this purpose. The study was conducted manually by a trained researcher on MRI performed during the hospitalization. The images were processed in transverse T2-dependent MR using a graphic Wacom Bamboo Pen \& Touch tablet and the tools available in the program used to measure the area of the selected structure. T2-weighted axial slices were used to facilitate distinguishing between the muscle and the fat tissue. The sections were evaluated at half height of the intervertebral disc. Three measurements were performed and the mean value was calculated.

The method of measurements is presented in Figs. 2 and 3 . The results are given in $\mathrm{cm}^{2}$. The following parameters were measured:

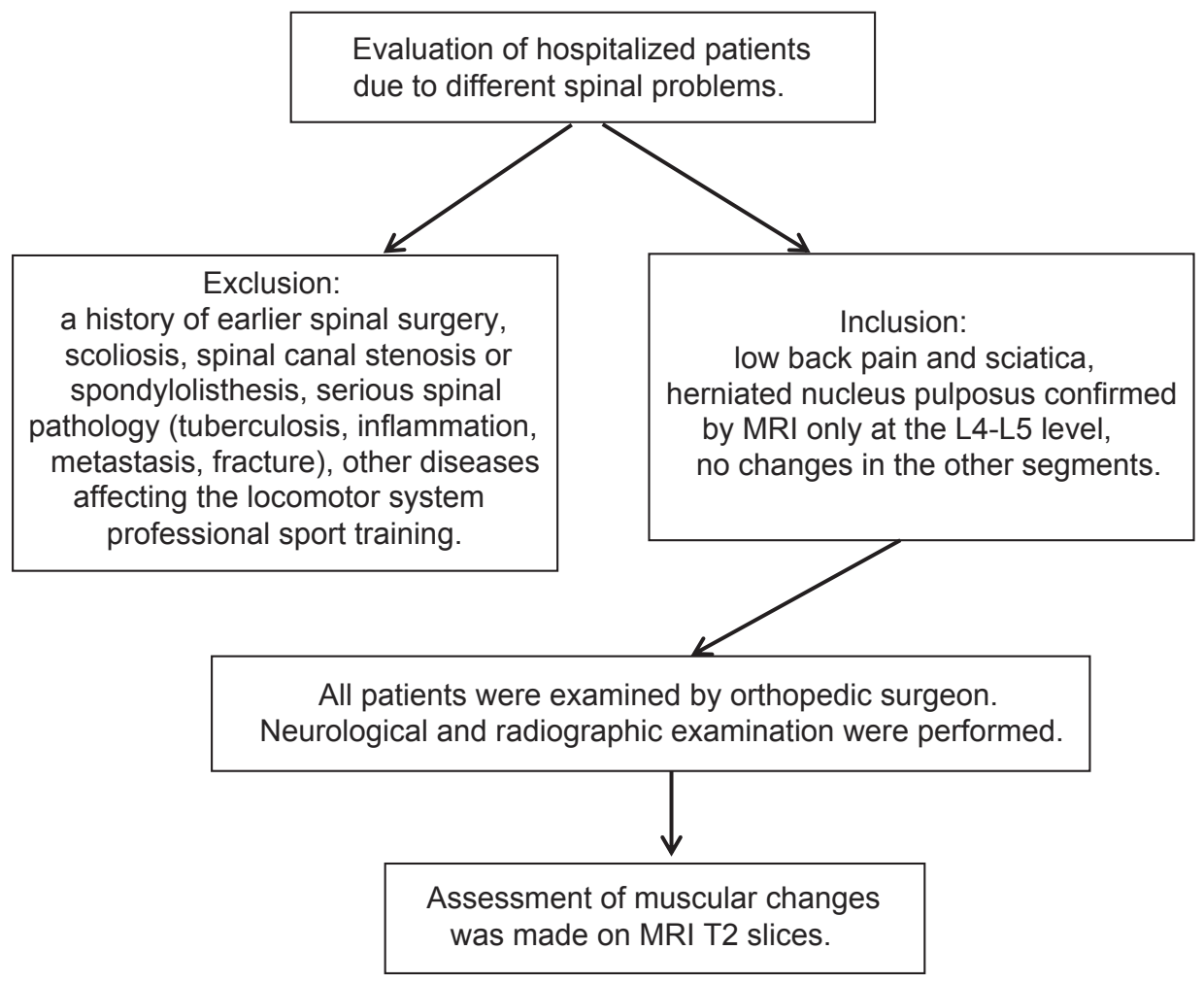

Fig. 1. Flow diagram regarding patients inclusion into the study 


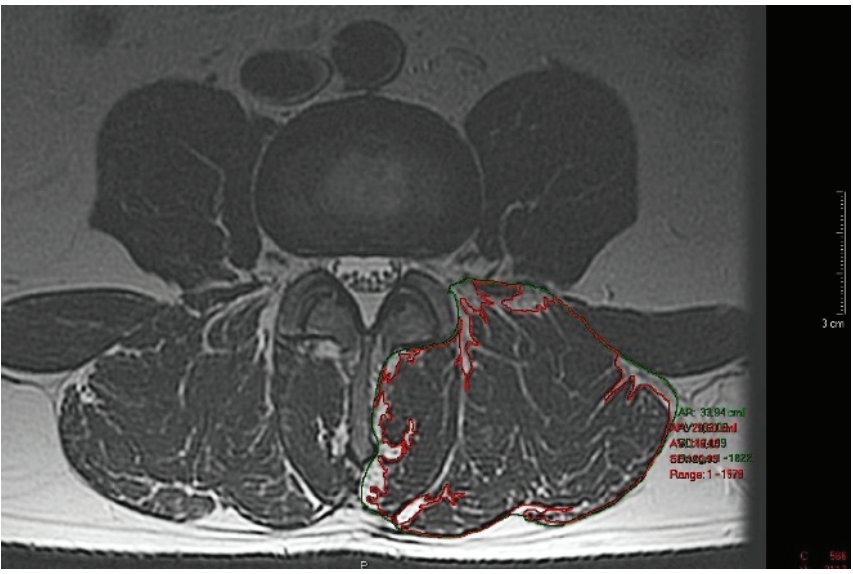

Fig. 2. Example of the cross-sectional area measurements taken from a T2 weighted axial slice from the healthy L3-L4 level

1. CSA of the entire spine extensor muscles,

2. CSA of the muscle tissue (CSA-M) of the spine extensor muscles for the right side (R) and for the left side (L),

3. CSA of the fat tissue (CSA-F) of the spine extensor muscles for the right and left side at the L3-L4 and L4-L5 levels.

We decided not to distinguish the ipsi- or contralateral side of the disc extrusion due to the fact that in most cases it had a central component due to its size (Fig. 3).

\section{Statistical analysis}

For all of the investigated variables, mean values and standard deviations for both levels were calculated and a comparison was made of the levels using Student's t-test for dependent samples (Table 1).

\section{Results}

The results were used to verify the hypothesis concerning the significance of the differences at the L3-L4 level with an intact intervertebral disc, as compared to the level of L4-L5 with disc extrusion. The areas of the entire extensor muscle CSA at the level of L3-L4 and L4-L5 were similar. The comparison of the results for the total crosssection of the entire extensor muscle CSA, the CSA-M size and the cross-sectional adipose tissue area (CSA-F) at the levels of L3-L4 and L4-L5 on the right and left side are summarized in Table 1.

It was decided to calculate the ratio of the CSA-F of the fat tissue to the CSA of the entire extensor muscle tissue. We did not find significant differences at the level of the intact intervertebral disc $(10.46 \pm 10.91$ on the right side; $10.82 \pm 9.89$ on the left side) or at the level of the herniated nucleus pulposus $(34.51 \pm 23.14$ on the right side; $28.93 \pm 20.45$ on the left side). These differences

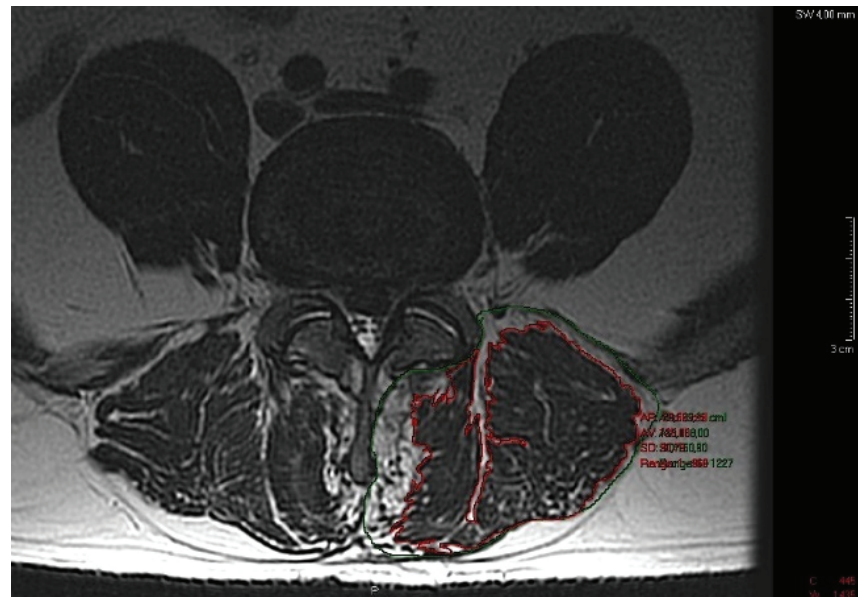

Fig. 3. Example of the cross-sectional area measurements taken from a T2 weighted axial slice at L4-L5 level with disc extrusion

between the right and left side were not statistically significant, but there was a highly significant difference between the levels.

At the healthy level, the ratio of CSA-F to CSA averaged $8.84 \pm 6.68$ on the right side, and $9.20 \pm 6.41$ on the left side. On the other hand, at the level with the herniation, this ratio was significantly higher and equaled an average of $24.05 \pm 9.87$ for the right side and $21.09 \pm 8.99$ for the left side.

Statistically insignificant differences were found comparing the CSA-M at L3-L4 and L4-L5 levels. All other differences proved to be highly statistically significant - at the level of $p<0.001$. The CSA-M at the healthy L3-L4 level on either side was significantly greater than that at the level of L4-L5 with disc extrusion. In contrast, the CSA of the fat tissue at the L3-L4 level was significantly lower than that at the L4-L5 level (Table 1).

\section{Discussion}

The study confirmed that in patients with disc disease there were evident signs of muscle tissue infiltration by adipose tissue - at the level with disc extrusion - compared to the healthy intervertebral disc. It is likely that the changes occur due to reduced activity of the muscles, whose tension causes compressive forces acting on the intervertebral disc. The penetration of the muscle tissue by fat tissue is not caused by a generalized limitation of the patients' functioning, because at the adjacent level with a healthy intervertebral disc the degree of adipose tissue infiltration was significantly smaller.

Studies on the atrophic changes leading to infiltration of the muscle tissue with adipose tissue because of pain and inactivity are not numerous. The analysis of the significance of the differences at the level of L3-L4 with an 
Table 1. Comparison of the entire cross-sectional muscle areas at the level of L3-L4 and L4-L5 for the total CSA of the multifidus, the ratio of CSA of the muscle tissue and the CSA of the adipose tissue

\begin{tabular}{lccc}
\hline & Mean & SD & p value \\
\hline CSA R on L3- L4 in cm $^{2}$ & 25.79 & 5.49 & 0.788 \\
CSA R on L4-L5 in cm & 25.64 & 5.14 & \\
CSA L on L3-L4 in cm ${ }^{2}$ & 25.83 & 5.42 & 0.050 \\
CSA L on L4-L5 in cm ${ }^{2}$ & 24.75 & 5.25 & \\
CSA -M R on L3-L4 in cm² & 23.55 & 5.49 & $<.001$ \\
CSA -M R on L4-L5 in cm ${ }^{2}$ & 19.57 & 5.20 & \\
CSA -M L on L3- L4 in cm ${ }^{2}$ & 23.52 & 5.53 & $<.001$ \\
CSA -M L on L4-L5 in cm cm $^{2}$ & 19.61 & 5.23 & \\
CSA -F R on L3-L4 in cm & 2.25 & 1.67 & $<.001$ \\
CSA -F R on L4-L5 in cm ${ }^{2}$ & 6.07 & 2.62 & \\
CSA -F L L3-L4 in cm & 2.31 & 1.58 & $<.001$ \\
CSA -F L L4-L5 in cm ${ }^{2}$ & 5.14 & 2.32 & \\
Ratio fat/ muscle tissue R P L3-L4 & 10.46 & 10.91 & $<.001$ \\
Ratio fat/ muscle tissue R L4-L5 & 34.51 & 23.14 & \\
Ratio fat/ muscle tissue L L3-L4 & 10.81 & 9.89 & $<.001$ \\
Ratio fat/ muscle tissue L L4-L5 & 28.93 & 20.45 & \\
Ratio fat/ CSA R L3-L4 & 8.84 & 6.68 & $<.001$ \\
Ratio fat/ CSA R L4-L5 & 24.05 & 9.87 & \\
Ratio fat/ CSA L L3-L4 & 9.20 & 6.41 & $<.001$ \\
Ratio fat/ CSA L L4-L5 & 21.09 & 8.99 & \\
\hline
\end{tabular}

Paraspinal muscles cross-sectional areas (CSA), R-right, L-left

intact intervertebral disc compared to the level of L4-L5 with the herniated nucleus pulposus proved to be statistically insignificant in terms of the total muscle area for the right and the left side. This is consistent with the previous results presented by Ranson and Ropponen [19, 21]. Similar results were also obtained by Danneels et al. [6]. They studied the total CSA of the paraspinal muscles, the multifidus and psoas, at three levels of the lumbar spine. The group with chronic lumbar pain was compared to the control group without back pain. In the analysis of the fat tissue area, no significant differences were found between the two groups for any of the three examined muscles. The authors came to the conclusion, however, that an etiologic relationship between the multifidus muscle hypotrophy and the occurrence of low back pain (LBP) should not be ruled out. There may also be an inverse relationship muscular hypotrophy may be a consequence of LBP. The pain can lead to inhibition of the multifidus muscle activity [11]. The proper function of this muscle is important because of its role in the stabilization of the lumbar spine, and its disappearance may be a reason for the high proportion of recurrent LBP.
Chan et al. observed different patterns of change in the flexibility and the CSA of the multifidus muscle depending on the adopted posture. Patients with chronic LBP, compared to the control group, had a smaller CSA of the multifidus muscles. These muscles showed greater stiffness in response to physiological static load [4].

Ali et al. found that adipose tissue infiltration was significantly greater in patients with spinal stenosis compared to patients affected by discopathy. The duration of symptoms probably played an important role [1]. In our study, patients with spinal stenosis were not examined, but fat tissue infiltration found at the level of herniation was significantly higher than that at the healthy level.

Barker et al. observed that CSA of paraspinal muscles correlated with duration of symptoms [2].

Goubert et al. found a higher amount of fat infiltration in the lumbar muscles (multifidus and extensors), in the absence of clear atrophy, in continuous chronic low back pain patients compared with non-continuous and recurrent low back pain patients. Their results underline the differences in muscle structure and muscle function between different LBP populations [9]. 
Shahidi et al. evaluated quantitatively multifidus tissue composition and structure, inflammation, vascularity, and degeneration in individuals with chronic degenerative lumbar spine pathology. Multifidus muscles biopsies were acquired from patients undergoing surgery. They concluded that changes in muscle tissue are more complex than simple atrophy including on average $48.5 \%$ muscle, $11.7 \%$ adipose tissue, and $26.1 \%$ collagen tissue, elevated inflammatory cells and decreased vascularity [22].

Guo et al. observed similar changes - paravertebral muscle degeneration in degenerative lumbar instability measured at L4-L5 and L5-S1 levels and compared to the control group [10].

Kjarer et al. found that ingrowth of adipose tissue was associated with age of patients suffering from lumbar spine pain, but this relationship did not exist among young people [13]. In the analyzed material, these dependencies were not correlated.

Lee et al. stated that in 21 analyzed women affected by the loss of lordosis, there was significantly greater fat tissue infiltration of paraspinal muscles compared to the control group [14]. The researchers also analyzed the isometric performance of the multifidus. They examined the CSA and the ability to perform a voluntary contraction using ultrasound tests. The results of this study confirm the earlier findings showing that the pattern of the multifidus muscle hypotrophy in patients with chronic lumbar pain was local and not generalized [24]. Our study confirmed these findings.

Our results are supported by the results obtained by Beattie et al. These authors did not observe, based on the analysis of patients with back pain and sciatica lasting less than 6 weeks, any asymmetry at the level above the herniated nucleus pulposus, but they noted increased hypertrophy of the adipose tissue at the level of the hernia [3]. It should be remembered that healthy subjects had an asymmetric CSA of the paraspinal muscles on the right and on the left side. These variations were greater than $10 \%$ [18].

The value of the study. The results of this study showed segmental infiltration of the muscle tissue with the fat tissue, occurring only at the level of the damaged intervertebral disc. The study included a homogeneous group of patients with single-level disc extrusion and sciatica qualified for surgical treatment.

Limitations of the study. We could not compare the study group results with a healthy control group due to high costs of MRI examinations and the ethical issue of conducting MRI on asymptomatic people.

\section{Conclusions}

1. At the level of painful herniated nucleus pulpous, there occurs significant ingrowth of the adipose tissue into the muscle tissue of the multifidus and short rotator muscles.
2. These changes were not observed at an adjacent level with an intact intervertebral disc.

3.It seems reasonable to introduce isolated strengthening exercises of these muscles after the resolution of pain in order to rebuild spine function and stability.

Conflict of interest: Authors state no conflict of interest.

\section{References}

1. Ali I., Ulbricht C., McGregor A.H. (2011) Degeneration of the extensor muscle group in a surgical low back and leg pain population. J. Back Musculoskelet. Rehabil., 24(1): 23-30.

2. Barker K.L., Shamley D.R., Jackson D. (2004) Changes in the cross-sectional area of multifidus and psoas in patients with unilateral back pain: the relationship to pain and disability. Spine (Phila Pa 1976); 15; 29(22): E515-9.

3. Battié M.C., Niemelainen R., Gibbons L.E., Dhillon S. (2012) CIs level- and side-specific multifidus asymmetry a marker for lumbar disc pathology? Spine J., 12(10): 932-939.

4. Chan S.T., Fung P.K., Ng N.Y., Ngan T.L., Chong M.Y., Tang C.N., He J.F., Zheng Y.P. (2012) Dynamic changes of elasticity, cross-sectional area, and fat infiltration of multifidus at different postures in men with chronic low back pain. Spine J., 12(5): 381-388.

5. Chen J.Y., Ding Y., Lv R.Y., Liu Q.Y., Huang J.B., Yang Z.H., Liu S.L. (2011) Correlation between MR imaging and discography with provocative concordant pain in patients with low back pain. Clin. J. Pain, 27(2): 125-130.

6. Danneels L.A., Vanderstraeten G.G., Cambier D.C., Witvrouw E.E., De Cuyper H.J. (2000) CT imaging of trunk muscles in chronic low back pain patients and healthy control subjects. Eur. Spine J., 9(4): 266-272.

7. DePalma M.J., Ketchum J.M., Saullo T. (2011). What is the source of chronic low back pain and does age play a role? Pain Med., 12(2): 224-233.

8. Gille O., Jolivet E., Dousset V., Degrise C., Obeid I., Vital J.M., Skalli W. (2007) Erector spinae muscle changes on magnetic resonance imaging following lumbar surgery through a posterior approach. Spine, 32(11): 1236-1241.

9. Goubert D., De Pauw R., Meeus M., Willems T., Cagnie B., Schouppe S., Van Oosterwijck J., Dhondt E., Danneels L. (2017) Lumbar muscle structure and function in chronic versus recurrent low back pain: a cross-sectional study. Spine J., 17(9): 1285-1296. DOI: 10.1016/j. spinee.2017.04.025. Epub 2017 Apr 26.

10. Guo X., Zhang X., Ding W., Yang D., Ma L., Xie D., Wang H., Wang H., Lu K., Yang S. (2014) Imaging study 
of paravertebral muscle degeneration in degenerative lumbar instability. Zhonghua Wai Ke Za Zhi.; 52(8): 571-575.

11. Hodges P., Holm A.K., Hansson T., Holm S. (2006) Rapid atrophy of the lumbar multifidus follows experimental disc or nerve root injury. Spine (Phila Pa 1976).;1, 31(25): 2926-2933.

12. Kader D.F., Wardlaw D., Smith F.W. (2000) Correlation between the MRI changes in the lumbar multifidus muscles and leg pain. Clin. Radiol., 55(2): 145-149

13. Kjaer P., Bendix T., Sorensen J.S., Korsholm L., Leboeuf-Yde C. (2007) Are MRI-defined fat infiltrations in the multifidus muscles associated with low back pain? BMC Med., 5: 2.

14. Lee J.C., Cha J.G., Kim Y., Kim Y.I., Shin B.J. (2008) Quantitative analysis of back muscle degeneration in the patients with the degenerative lumbar flat back using a digital image analysis: comparison with the normal controls Spine (Phila Pa 1976)., 1, 33(3): 318-325. DOI: 10.1097/BRS.0b013e318162458f.

15. Maniadakis N., Gray A. (2000) The economic burden of back pain in the UK. Pain, 84(1): 95-103.

16. Mannion A.F., Käser L., Weber E., Rhyner A., Dvorak J., Müntener M. (2000) Influence of age and duration of symptoms on fibre type distribution and size of the back muscles in chronic low back pain patients. Eur. Spine J., 9(4): 273-281.

17. Mengiardi B., Schmid M.R., Boos N., Pfirrmann C.W., Brunner F., Elfering A., Hodler J. (2006) Fat content of lumbar paraspinal muscles in patients with chronic low back pain and in asymptomatic volunteers: quantification with MR spectroscopy. Radiology, 240(3): 786-792.

18. Niemeläinen R., Briand M.M., Battié M.C. (2011) Substantial asymmetry in paraspinal muscle cross-sectional area in healthy adults questions its value as a marker of low back pain and pathology. Spine (Phila Pa 1976), 1; 36(25): 2152-2157.
19. Ranson C.A., Burnett A.F., Kerslake R., Batt M.E., O'Sullivan P.B. (2006) An investigation into the use of MR imaging to determine the functional cross sectional area of lumbar paraspinal muscles, Eur. Spine J., 15(6): 764-773.

20. Rintala H., Sovelius R., Rintala P., Huhtala H., Siitonen S., Kyröläinen H. (2017) MRI findings and physical performance as predictors of flight-induced musculoskeletal pain incidence among fighter pilots. Biomed. Hum. Kinet., 9, 133-139. DOI: 10.1515/bhk-2017-0019.

21. Ropponen A., Videman T., Battié M.C. (2008) The reliability of paraspinal muscles composition measurements using routine spine MRI and their association with back function. Man. Ther., 13(4): 349-356.

22. Shahidi B., Hubbard J.C., Gibbons M.C., Ruoss S., Zlomislic V., Allen R.T., Garfin S.R., Ward S.R. (2017) Lumbar multifidus muscle degenerates in individuals with chronic degenerative lumbar spine pathology. J. Orthop. Res., 8. DOI: 10.1002/jor.23597. [Epub ahead of print].

23. Smith M., Davis M.A., Stano M., Whedon J.M. (2013) Aging baby boomers and the rising cost of chronic back pain: secular trend analysis of longitudinal Medical Expenditures Panel Survey data for years 2000 to 2007. J. Manipulative Physiol. Ther., 36(1): 2-11. DOI: 10.1016/j. jmpt.2012.12.001.

24. Wallwork T.L., Stanton W.R., Freke M., Hides J.A. (2009) The effect of chronic low back pain on size and contraction of the lumbar multifidus muscle. Man Ther., 14(5): 496-500.

\section{Received 07.11.2017 \\ Accepted 11.01.2018}

(C) University of Physical Education, Warsaw, Poland 